www.nature.com/ejhg

\title{
Hereditary spastic paraplegia caused by mutations in the SPG4 gene
}

\author{
Joachim Bürger ${ }^{1}$, Nuria Fonknechten ${ }^{2}$, Maria Hoeltzenbein ${ }^{3}$, Luitgart Neumann ${ }^{1}$, \\ Elfriede Bratanoff ${ }^{4}$, Jamilé Hazan ${ }^{2}$ and André Reis ${ }^{1,5}$
}

\author{
${ }^{1}$ Institute of Human Genetics, Charité, Humboldt-Universität, Berlin, Germany; ${ }^{2}$ Genoscope, Evry, France; ${ }^{3}$ Institute \\ of Human Genetics, Ernst-M oritz-Arndt-Universität, Greifswald; ${ }^{4}$ Sozial pädiatrisches Zentrum, Erfurt; ${ }^{5}$ M olecular \\ Genetics and Microsatellite Centre, Max-D elbrück-Centre, Berlin-Buch, Germany
}

\begin{abstract}
Autosomal dominant hereditary spastic paraplegia (AD-HSP) is a genetically heterogeneous neurodegenerative disorder characterised by progressive spasticity of the lower limbs. The SPG4 locus at 2p21-p22 accounts for $40-50 \%$ of all AD-HSP families. The SPG 4 gene was recently identified. It is ubiquitously expressed in adult and foetal tissues and encodes spastin, an ATPase of the AAA family. We have now identified four novel SPG4 mutations in German AD-HSP families, including one large family for which anticipation had been proposed. Mutations include one frame-shift and one missense mutation, both affecting the Walker motif B. Two further mutations affect two donor splice sites in introns 12 and 16 , respectively. RT-PCR analysis of both donor splice site mutations revealed exon skipping and reduced stability of aberrantly spliced SPG4 mRNA. All mutations are predicted to cause loss of functional protein. In conclusion, we confirm in German families that SPG4 mutations cause AD-HSP. Our data suggest that SPG4 mutations exert their dominant effect not by gain of function but by haploinsufficiency. If a threshold level of spastin were critical for axonal preservation, such threshold dosage effects might explain the variable expressivity and incomplete penetrance of SPG4-linked AD-HSP. European Journal of Human Genetics (2000) 8, 771-776.
\end{abstract}

Keywords: Strumpell's disease; spastic paraplegia; SPG4; spastin; splice mutation; haploinsufficiency; dominant negative effect

\section{Introduction}

Spastic paraplegias are a genetically heterogeneous group of diseases that share a defect of the axons of the pyramidal tracts. Both, corticospinal tracts and dorsal column fibres are affected by degeneration. The universal clinical sign of the disease is progressive weakness and spasticity of the lower limbs. ${ }^{1}$ Among the hereditary spastic paraplegias (HSP), pure and complicated forms have been described and autosomal recessive, autosomal dominant and $\mathrm{X}$-linked patterns of inheritance reported. ${ }^{1}$

Several types of HSP have been mapped to different loci, demonstrating extensive genetic heterogeneity. AD-HSP has been mapped to chromosome14q11.2-q24.3 (SPG3) in

Correspondence: Dr Joachim Bürger, Institut für Humangenetik, Campus Virchow-Klinikum, Charité, Augustenburger Platz 1,

13353 Berlin, Germany. Tel: + 4930 45066328; Fax: + 4930 45066904; E-mail: joachim.buerger@charite.de

Received 5 January 2000; revised 19 April 2000; accepted 30 May 2000
French, American, German and Tibetan families, ${ }^{2-5}$ to chromosome2p21-p24 (SPG4) in French, Dutch, North American, Tunisian, Belgian and German families, ${ }^{3,6-8}$ to $15 q$ (SPG6) in one family of Irish descent, ${ }^{9}$ to $8 q 23-24$ (SPG8) in a North American kindred of German descent and in an English family ${ }^{10,11}$ and to $12 \mathrm{q} 13$ (SPG10) in an English family. ${ }^{12}$ There must be at least one further AD-HSP locus, since in one family linkage to all these five loci has been excluded. ${ }^{12}$

Cloning of mutant genes identified some of the aetiologies involved in HSP. X-linked recessive HSP appears to be caused by mutant $P L P^{13}$ that is required in the terminal stages of myelin compaction. The LICAM gene is mutated in complicated $\mathrm{X}$-linked recessive HSP. ${ }^{14}$ LICAM is involved in neuronneuron adhesion and neuronal cell migration. In autosomal recessive HSP, mutant paraplegin has been identified, which is highly homologous to yeast mitochondrial ATPases. ${ }^{15}$ 
Immunofluorescence analysis showed that paraplegin localises to mitochondria and muscle biopsies from patients with paraplegin mutations showed mitochondrial OXPHOS defects, thus suggesting a mechanism for neurodegeneration.

The first gene that is mutated in AD-HSP patients, SPG4, was identified very recently. ${ }^{16}$ Its open reading frame encodes a protein, spastin, of 616 amino acids. The $C$-terminus shows high homology to AAA proteins. The AAA cassette ${ }^{17}$ is localised between amino acids 342 and 599 in the spastin sequence. The three conserved ATPase domains, including Walker motifs $A$ and $B^{18}$ and the AAA minimal consensus, were located in the AAA cassette between amino acid positions382-389, 437-442 and 480-498, respectively. Walker motif A, al so called p-loop, which corresponds to the ATP-binding domain, and Walker motif $B$ are highly conserved between spastin and all AAA family members. The AAA protein family is a group of ATPases with diverse functions. Spastin is probably involved in the assembly or function of nuclear protein complexes. ${ }^{16}$

We have analysed the SPG4 gene in five German AD-HSP families and one HSP patient without family history to investigate whether this gene might also be affected in patients other than the original sample. Additionally, we have addressed the frequency and type of SPG 4 variants and their predicted functional consequences for the peptide with respect to the clinical phenotype. Here we present four novel mutations in the SPG 4 gene, one missense, one frame-shift and two splice mutations that were both shown to cause exon skipping.

\section{Materials and methods Patients}

We analysed six independent index cases with spastic paraplegia. All of them are of German origin. Patient 1 is from the original family in whom we previously reported linkage to the SPG4 locus and evidence for anticipation. ${ }^{8}$ All patients were seen and investigated clinical ly by experienced neurologists. The age of onset varied widely from 1 to 55 years. None of the patients had a complicated form of HSP. Two obligate carriers from families 1 and 3 considered themselves unaffected, despite presenting mild signs on neurological investigation. Five of six patients showed autosomal dominant inheritance of the disorder. The sixth case is a singleton, but it is not known whether the father was affected. After informed consent was obtained, high molecular weight genomic DNA was prepared from peripheral blood leukocytes of AD-HSP patients and normal controls by standard methods.

\section{Linkage analysis}

$M$ icrosatellite genotypes were determined at the AD-HSP Ioci SPG3, SPG4 and SPG6. In larger families, two-point linkage data were calculated with the LINKAGE program package, version 5.10 .

\section{PCR}

The genomic DNA sequence of the human SPG4 gene has been determined, ${ }^{16}$ permitting the design of PCR primers from intronic sequences. PCR and sequencing primers were those used in the previous study ${ }^{16}$ (http://www.genoscope.cns.fr) except for sequencing primers spa2c (TAGTGTACTCTTCATACGATCT), spa5c (GTITACAAATGTTGCTTGTC), spa10m (AGATAGTCTTTAAGCTTGCCC) and spa16c (CCTTCAACAATTTCAACTGC). PCR was performed with $20 \mathrm{ng}$ of DNA, $10 \mathrm{~mm}$ Tris, $50 \mathrm{~mm} \mathrm{KCl}, 1.5 \mathrm{~mm} \mathrm{MgCl}_{2}$, $100 \mu \mathrm{m}$ of each dNTP, $1 \mu \mathrm{m}$ of each primer and $0.5 \mathrm{u}$ Taq polymerase (PE Applied Biosystems, Weiterstadt, Germany) in a volume of $30 \mu \mathrm{l}$. PCR was carried out in GeneAmp 9600 thermo cyclers (PE Applied Biosystems), starting with an initial denaturation of $5 \mathrm{~min}$ at $94^{\circ} \mathrm{C}$ followed by 3 cycles at decreasing annealing temperatures. 30 cycles were run for $20 \mathrm{~s}$ at $94^{\circ} \mathrm{C}, 20 \mathrm{~s}$ at a primer-specific annealing temperature and $40 \mathrm{~s}$ at $72^{\circ} \mathrm{C}$ followed by a final elongation of $5 \mathrm{~min}$ at $72^{\circ} \mathrm{C}$.

\section{Sequencing}

PCR products were purified with Qiaquick PCR purification kit (Qiagen, Hilden, Germany). PCR fragments were sequenced directly or after cloning (TA Cloning Kit, Invitrogen, Groningen, The Netherlands) using Big-Dye-Terminator Cycle Sequencing Kit (PE Applied Biosystems). Sequencing reactions were purified with Dyeex Spin kit (Qiagen) and run and analysed on an ABI Prism 310 Genetic Analyzer (PE Applied Biosystems).

\section{Restriction enzyme analysis}

Mutations D441G and $1853+1 \mathrm{G} \rightarrow \mathrm{T}$ were analysed by $\mathrm{Hphl}$ and $\mathrm{Mnll}$ restriction enzymes (New England Biolabs, Frankfurt, Germany) respectively. PCR products spalla/spal2m and $\mathrm{spa16} / \mathrm{spa16m}$ were digested according to the manufacturer's conditions and separated on $2 \%$ agarose gels.

\section{RT-PCR}

In families with mutations that could affect splicing, total RNA from blood lymphocytes was extracted from affected individuals and controls using Trizol (Life Technologies, Karlsruhe, Germany). cDNA synthesis was performed by incubating approximately $1 \mu \mathrm{g}$ RNA, $100 \mathrm{pmol}$ random hexamer primers (Pharmacia, Freiburg, Germany) and $200 \mathrm{u}$ Superscript II (Life Technologies) according to standard procedures. Since the mutations under investigation potentially affected exons11, 12 and 16, we carried out a PCR which generated a fragment from exon 10 to 17, using primers spaC10 (TGGGAGAAGGAGAGAAATTG) and spaC17 (CAAGGATTCAGTGAAGTCAG). PCR was performed with $1 / 5$ th of the CDNA preparation $(4 \mu \mathrm{l}), 10 \mathrm{~mm}$ Tris, $50 \mathrm{~mm} \mathrm{KCl}$, $1.5 \mathrm{~mm} \mathrm{MgCl}_{2}, 100 \mu \mathrm{m}$ of each dNTP, $1 \mu \mathrm{m}$ of each primer and 
0.5u Taq polymerase (PE Applied Biosystems) in a volume of $50 \mu \mathrm{l}$ in a GeneAmp 9600 thermocycler (PE Applied Biosystems), starting with an initial denaturation of $5 \mathrm{~min}$ at $94^{\circ} \mathrm{C}$ followed by 4 cycles at annealing temperatures, reducing from $63^{\circ} \mathrm{C}$ to $57^{\circ} \mathrm{C}$. Thereafter $30 \mathrm{cycles}$ were run for $15 \mathrm{~s}$ at $93^{\circ} \mathrm{C}, 15 \mathrm{~s}$ at $55^{\circ} \mathrm{C}$ and $45 \mathrm{~s}$ at $72^{\circ} \mathrm{C}$. The final elongation at $72^{\circ} \mathrm{C}$ was $5 \mathrm{~min}$. PCR products were separated on $2 \%$ agarose gels in $1 \times$ TAE buffer. Individual bands were visualised on a long-wavelength UV transilluminator and excised by using sterile scal pels. Gel slices were centrifuged through UItrafreeDA columns (Millipore, Eschborn, Germany) and diluted in $500 \mu \mathrm{l} \mathrm{H}_{2} \mathrm{O}$. $1 \mu \mathrm{l}$ was then re-amplified and sequenced as described above

\section{Results}

Initially, we investigated all families for linkage to the SPG4 locus. Within the five families, affected persons shared a common microsatellite marker haplotype but only family 1 gave a significant lod score $(4.02$ at $\Theta=0.00)$. Subsequently, we investigated these five unrelated families with AD-HSP and one singleton patient with spastic paraplegia for mutations in the SPG4 gene. We identified four different mutations that have not been described before (Table1).

In family 1 , direct sequencing of the PCR product corresponding to exon 11 revealed heterozygosity for an $A$ to $G$ substitution of the first nucleotide of this exon. This alteration causes an aspartic acid to glycine exchange (D441G) in the Walker B motif of the peptide. Within this motif of six amino acids (IIFIDE), the fifth position is $D$ (aspartic acid) in eight of eight proteins that exhibit high homology to spastin. ${ }^{16}$ Aspartic acid is very polar and thus nearly always found on the outside of proteins, whereas glycine is non-polar and tends to be on the inside. Consequently, strong effects of aspartic acid to glycine mutations are also described in several other genes, eg in the fibrillin 1 gene, where it causes a severe form of Marfan syndrome. ${ }^{19}$ Moreover, D441G creates a new restriction enzyme site for $\mathrm{H}$ phl. Therefore, we confirmed the mutation in the index patient by $\mathrm{Hphl}$ digestion of the PCR product and also showed co-segregation of the mutation with the phenotype in this family (Figure1). Since mutations of the first or last

Table 1 Novel mutations in the SPG 4 gene in patients with AD-HSP

\begin{tabular}{lll}
\hline Mutation & Site & Consequence \\
\hline $1340-1344$ del & Exon 9 & $\begin{array}{l}\text { Frameshift codons 405-440, } \\
\text { Stop at 440 }\end{array}$ \\
$1447 \mathrm{~A} \rightarrow \mathrm{G}$ & $\begin{array}{l}\text { Exon 11 } \\
\text { Exon and } \\
\text { I617-1618+2del } \\
\text { Intron 12 }\end{array}$ & $\begin{array}{l}\text { Donor splice site deletion } \\
\text { skipping of exons 11-12 and 11-13 } \\
\text { unstable mRNA } \\
\text { Donor splice site mutation } \\
\text { skipping of exon 16 } \\
\text { unstable mRNA }\end{array}$ \\
\hline
\end{tabular}

nucleotide in the exon were suggested to affect splicing, ${ }^{20}$ we extracted lymphocyte RNA and performed an RT-PCR that spans exons10-17. However, we only obtained the $521 \mathrm{bp}$ band expected, but no aberrant bands.

In family 3, direct sequencing of exon 16 PCR product showed heterozygosity for a $G$ to $T$ mutation in the donor splice site in intron 16, $1853+1 \mathrm{~g} \rightarrow \mathrm{t}$. Since this mutation destroys an Mnll site, digestion of exon 16 PCR product with $M$ nll was used to confirm the co-segregation of mutation and disorder. To investigate the effect of $1853+1 \mathrm{~g} \rightarrow \mathrm{t}$ on SPG4 splicing, we amplified an RT-PCR product that spans exons 10 to 17 from lymphocyte RNA. In addition to the expected band another smaller and very weak band was seen on agarose gels (Figure2). After excision from the gel and re-amplification, the aberrant band was sequenced from both directions, thereby demonstrating skipping of exon 16 (Figure 3). This observation is in accordance with expectations since mutations in the donor splice site predominantly cause skipping of the adjacent exon. ${ }^{21}$ The $1853+1 \mathrm{~g} \rightarrow \mathrm{t}$ mRNA bears a frame shift and a premature termination signal at codon 570. If translated, a spastin with erroneous amino acids at codons563-569 would result. The functionally important structures, the Walker A and B motifs and the AAA minimal consensus sequence, would not be affected by this mutation. However, our RT-PCR data show that the dose of

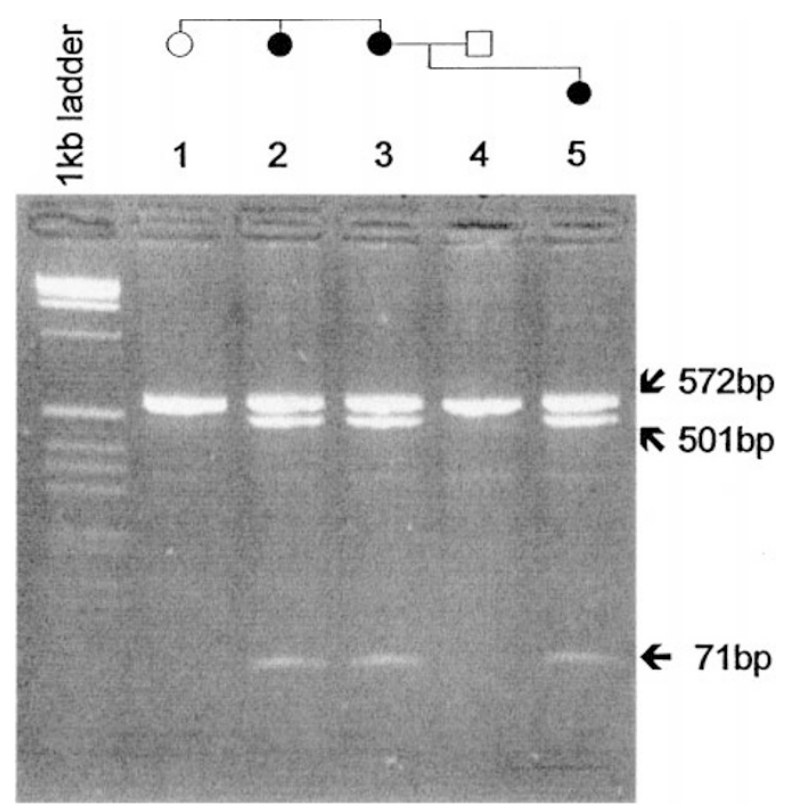

Figure 1 SPG4 mutation D441G in family 1. A heterozygous missense mutation creates an $\mathrm{Hphl}$ site. Restriction digestion with $\mathrm{Hphl}$ shows two digestion products of $501 \mathrm{bp}$ and $71 \mathrm{bp}$ in addition to the undigested $572 \mathrm{bp}$ fragment in affected individuals of family 1 . Unaffected persons show only an undigested $572 \mathrm{bp}$ PCR product. $1 \mathrm{~kb}$ ladder is used as size standard. 
the mutant mRNA is drastically reduced. We estimate the ratio of normal to mutant mRNA at approximately 100 to 1 .

In family 4, direct sequencing of the PCR product corresponding to exon 12 revealed heterozygosity for an AGgt deletion that affects two nucleotides of exon 12 and two nucleotides of the donor splice site in intron 12 . To analyse the effect of this 1617-1618+2del mutation on SPG4 splic-

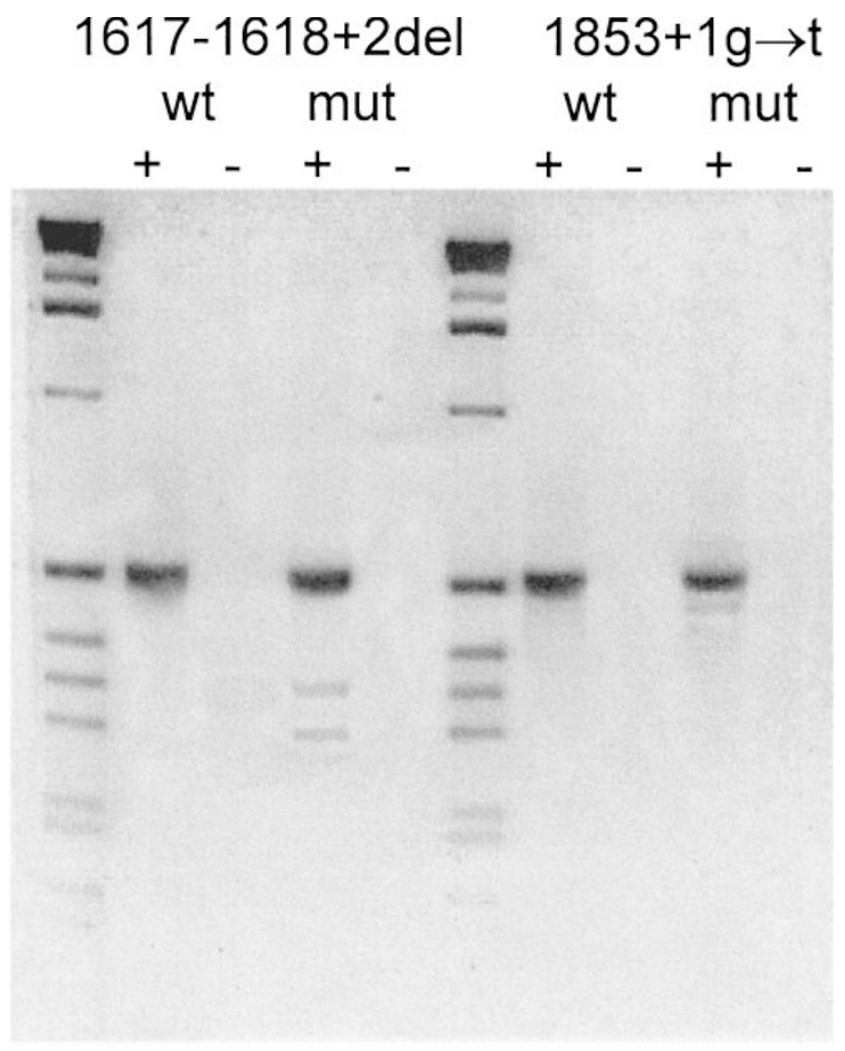

Figure 2 RT-PCR in AD-HSP patients with splice mutations. 1617-1618 +2del showed two weak aberrant bands and $1853+1 \mathrm{~g} \rightarrow \mathrm{t}$ one additional faint band. wt: wild-type; mut: mutant; + and -: with and without reverse transcriptase.

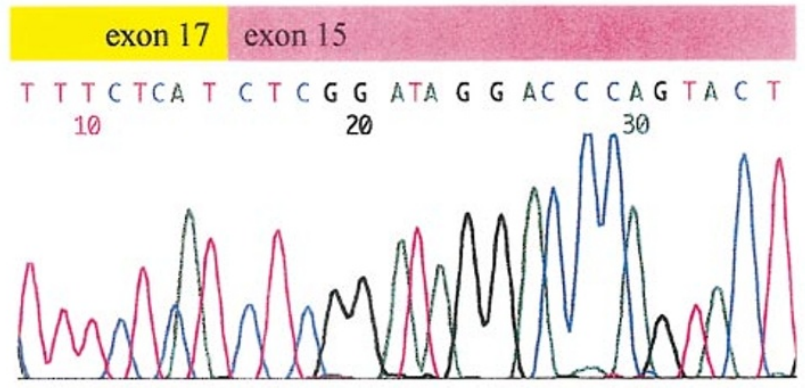

Figure 3 SPG 4 mutation $1853+1 \mathrm{~g} \rightarrow$ t in family 3 . Sequencing of single RT-PCR products showed that exon 15 is immediately followed by exon 17. This missense mutation in the donor splice site of exon 16 results in exon 16 skipping. ing, we performed an RT-PCR that spans exons10-17. In addition to the $521 \mathrm{bp}$ band of the wild type allele, we obtained smaller and very weak bands (Figure2). These were excised from the gel, reamplified and sequenced from both directions. We identified two aberrantly spliced mRNAs, one showing skipping of exons 11 and 12 and the other one missing exons11, 12 and 13 (Figure4). Mutations at splice sites often lead to the observation of multiple cDNA species after RT-PCR analysis. Sometimes mRNAs with multiple skipped exons are generated from the mutant DNA. In addition, splice mutations may exert a pleiotropic effect in that they lead to the generation of several differently spliced mRNAs. For example, a splice donor mutation in intron 5 of the HPRT geneled to (i) inclusion of intron 5, (ii) exclusion of exons 4 and 5 and (iii) exclusion of exons2-6. ${ }^{22}$ The 1617-1618+2del mRNAs without exons11-12 and without exons11-13 show frame shifts and premature termination signals at codons 472 and 447 respectively. If translated, spastin proteins with erroneous amino acids at codons441471 and 441-446 would result from this deletion. In both cases, the Walker B motif and the AAA minimal consensus sequence would not be present in such spastin. But, like the $1853+1 \mathrm{~g} \rightarrow \mathrm{t}$ mutation, the doses of mutant mRNAs are reduced by a factor of approximately 100 , which suggests that mutant SPG4 mRNAs are highly unstable.

In family 6 , direct sequencing of exon 9 PCR product revealed heterozygosity for a TATAA deletion in exon 9 ,
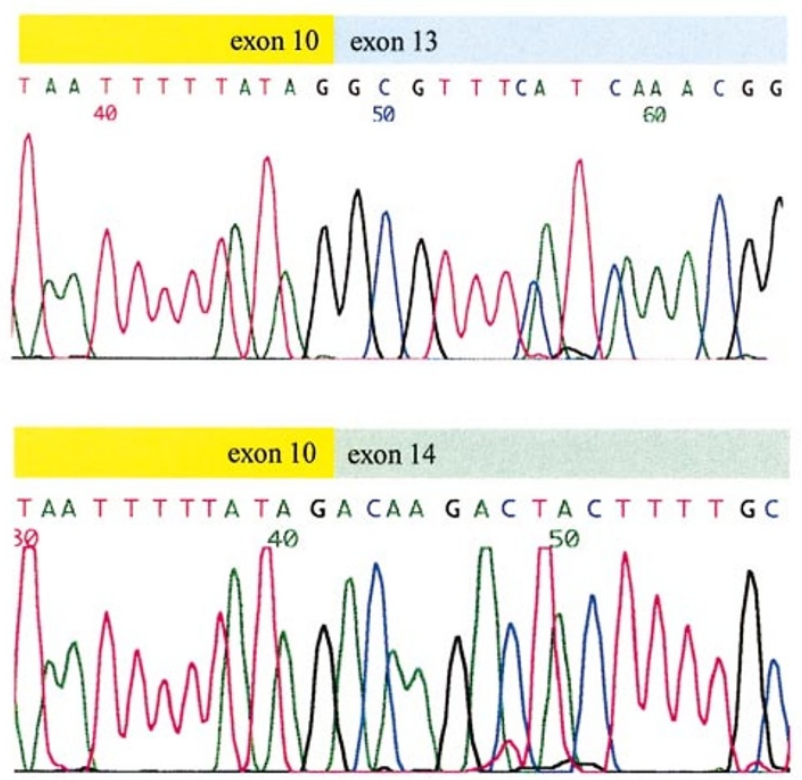

Figure 4 SPG4 mutation 1617-1618 +2del in family 4. Sequencing of single RT-PCR products showed that the sequence after exon 10 proceeds with either exon 13 or exon 14. This $4 \mathrm{bp}$ deletion that includes the donor splice site of exon 12 results in skipping of either exons $11-12$ or exons 11-13. 
1340-1344del. This mutation causes a frame shift in the SPG 4 open reading frame from codon 405 to 440 , where a premature termination codon is created. Such spastin protein would lack two of its functional domains, the Walker motif B and the AAA minimal consensus sequence.

The mutations D441G, 1340-1344del, 1617-1618+2del and $1853+1 \mathrm{~g} \rightarrow \mathrm{t}$ were not found on 180 chromosomes of normal individuals of German origin.

In family 2 and in singleton patient 5 , we did not find mutations in SPG4 exons1-17, the flanking intronic sequences and $200 \mathrm{bp}$ of the 5 ' untranslated region.

\section{Discussion}

Mutations on chromosome2p21-p22 cause approximately $40-50 \%$ of AD-HSP. The mutant gene at this locus, SPG4, was recently identified by positional cloning. ${ }^{16}$ Here, we describe four novel SPG4 mutations, thus confirming that they are relatively frequently found in AD-HSP patients.

No mutations were found in a patient without a family history and in a family where two definitely affected persons showed an identical haplotype, which was also present in a 23-year-old, so far unaffected person. We cannot exclude that large chromosomal re-arrangements might have occurred in the SPG 4 gene in family 2 and patient5. For example, the deletion of complete exons, including the adjacent intronic sequences, cannot be found by the approach we have used. These large deletions could be identified by Southern blotting, which is therefore a conceivable method for the further analysis of AD-HSP patients without 'small' SPG 4 mutations. Alternatively, mutations at other AD-HSP loci need to be considered.

\section{SPG4 mutations and their functional consequences}

The SPG4 mutation D441G destroys one of the most conserved sites of the protein, the Walker motif $B$ of the AAA cassette. The mutation 1340-1344del causes a spastin protein that would lack two of its three highly conserved functional domains, the Walker motif $B$ and the AAA minimal consensus sequence. The SPG 4 splice mutations $1853+1 \mathrm{~g} \rightarrow \mathrm{t}$ and 1617-1618+2del lead to exon skipping and drastically reduced doses of mutant mRNAs which suggests that they are highly unstable. Consequently, mutant spastin would be expressed, if at all, at extremely low levels and hence could not have a dominant negative effect (gain of function). Instead of this, our data support a haploinsufficiency mechanism as previously reported ${ }^{16}$ where spastin dosage would be a very critical factor for axon maintenance.

\section{Genotype/ phenotype correlation}

The intra-familial variability of severity and age of onset of the disease indicates that there cannot be a tight genotype/ phenotype correlation. We predicted severe functional disadvantages for all the four novel mutations described here, since they destroy highly conserved functional domains.
Moreover, we have shown that mutant SPG 4 mRNA is not stable. For these reasons, we would expect severe clinical effects in all families. In general, this is what we observed, but there are two individuals in families 1 and 3 who exhibit a significantly milder phenotype than their relatives with the same mutation. As discussed above, spastin dosage appears to be critical for axon maintenance. If it were to fall below a threshold level, axon preservation would fail. This model explains the clinical variability and also the reduced penetrance of SPG4-linked AD-HSP, especially if we postulate a dynamic threshold that interacts with other genetic and environmental factors.

\section{Exclusion of trinucleotide repeat expansion as cause of anticipation}

Anticipation was reported in one Dutch ${ }^{23}$ and six Danish ${ }^{24}$ AD-HSP families. We had clinically observed a striking decrease in the age of onset in family 1 of 10.8 years on average per transmitting meiosis. ${ }^{8}$ However, looking for expanded trinucleotide repeats by the repeat expansion detection method, ${ }^{25}$ we found that anticipation was not associated with expansion of repeats (Hatchwell and Bürger, unpublished). We show here, that this family anticipates a missense mutation in the SPG4 gene, D441G. Therefore we conclude that the clinically observed anticipation is due to an ascertainment bias. This was also demonstrated by clinical studies. $^{26,27}$

In conclusion, we present here four novel mutations in the SPG4 gene, one missense, one frame shift and two splice mutations. All mutations are predicted to cause loss of functional protein. Our data suggest that SPG 4 mutations exert their dominant effect not by gain of function, but by loss of spastin dosage. We hypothesise that a threshold level of spastin is critical for axonal preservation. This would explain the variable expressivity and incomplete penetrance of SPG4-linked AD-HSP. Moreover, increasing the expression of spastin might be a therapeutic option in the future. However, further studies on mutant spastin in AD-HSP patients as well as in an animal model are necessary to support our hypotheses.

\section{Acknowledgements}

We wish to thank Dr H M etzke, Institut für Humangenetik, Jena, the patients and their relatives for their co-operation. Two months after we submitted this manuscript, another SPG4 mutation report was published: Fonknechten N, Mavel D, Byrne P et al: Spectrum of SPG 4 mutations in autosomal dominant spastic paraplegia. Hum Mol Genet 2000; 9: 637-644.

\section{References}

1 McKusick VA: Online Mendelian Inheritance in Man. http:/ /www3.ncbi.nlm.nih.gov/omim/1999.

2 Hazan J, Lamy C, M elki J, Munnich A, de Recondo J, Weissenbach $\mathrm{J}$ : Autosomal dominant familial spastic paraplegia is genetically heterogeneous and one locus maps to chromosome14q. Nat Genet 1993; 5: 163-167. 
3 Hentati A, Pericak-Vance MA, Lennon F et al: Linkage of a locus for autosomal dominant familial spastic paraplegia to chromosome2p markers. Hum Mol Genet 1994; 3: 1867-1871.

4 Gispert S, Santos N, Damen R et al: Autosomal dominant familial spastic paraplegia: reduction of the FSP1 candidate region on chromosome $14 \mathrm{q}$ to $7 \mathrm{cM}$ and locus heterogeneity. Am J Hum Genet 1995; 56: 183-187.

5 Huang S, Zhuyu, Li H et al: Another pedigree with pure autosomal dominant spastic paraplegia (AD-FSP) from Tibet mapping to 14q11.2-q24.3. Hum Genet 1997; 100: 620-623.

6 Hazan J, Fontaine B, Bruyn RP et al: Linkage of a new locus for autosomal dominant familial spastic paraplegia to chromosome2p. Hum Mol Genet 1994; 3: 1569-1573.

7 De Jonghe $P$, Krols L, Michalik $A$ et al: Pure familial spastic paraplegia: clinical and genetic analysis of nine Belgian pedigrees. Eur J Hum Genet 1996; 4: 260-266.

8 Bürger J, Metzke H, Paternotte C, Schilling F, Hazan J, Reis A: Autosomal dominant spastic paraplegia with anticipation maps to a 4-cM interval on chromosome2p21-p24 in a large German family. Hum Genet 1996; 98: 371-375.

9 Fink JK, Wu CT, Jones SM et al: Autosomal dominant familial spastic paraplegia: tight linkage to chromosome15q. Am J Hum Genet 1995; 56: 188-192.

10 Hedera P, Rainier S, Alvarado D et al: Novel locus for autosomal dominant hereditary spastic paraplegia, on chromosome8q. Am J Hum Genet 1999; 64: 563-569.

11 Reid E, Dearlove AM, Whiteford ML, Rhodes M, Rubinsztein DC: Autosomal dominant spastic paraplegia: refined SPG8 locus and additional genetic heterogeneity. Neurology 1999; 53: 1844-1849.

12 Reid E, Dearlove AM, Rhodes M, Rubinsztein DC: A new locus for autosomal dominant 'pure' hereditary spastic paraplegia mapping to chromosome 12q13, and evidence for further genetic heterogeneity. Am J Hum Genet 1999; 65: 757-763.

13 Saugier-Veber $P$, Munnich A, Bonneau D et al: X-linked spastic paraplegia and Pelizaeus-Merzbacher disease are allelic disorders at the proteolipid protein locus. Nat Genet 1994; 6: 257-262.

14 Jouet $M$, Rosenthal $A$, Armstrong $G$ et al: X-linked spastic paraplegia (SPG1), MASA syndrome and X-linked hydrocephalus result from mutations in the L1 gene. Nat Genet 1994; 7: 402-407.

15 Casari G, De Fusco M, Ciarmatori S et al: Spastic paraplegia and OXPHOS impairment caused by mutations in paraplegin, a nuclear-encoded mitochondrial metalloprotease. Cell 1998; 93: 973-983.
16 Hazan J, Fonknechten N, Mavel D et al: Spastin, a new AAA protein, is altered in the most frequent form of autosomal dominant spastic paraplegia. Nat Genet 1999; 23: 296-303.

17 Beyer A: Sequence analysis of the AAA protein family. Protein Sci 1997; 6: 2043-2058.

18 Walker JE, Saraste MJ, Runswick JJ, Gay NJ: Distantly related sequences in the $\alpha$ - and $\beta$-subunits of ATPase, myosin, kinases and other ATP-requiring enzymes and a common nucleotide-binding fold. EMBO J 1982; 1: 945-951.

19 Putnam EA, Cho M, Zinn AB, Towbin JA, Byers PH, Milewicz DM: Delineation of the Marfan phenotype associated with mutations in exons23-32 of the FBN1 gene. Am J Med Genet 1996; 62: 233-242.

20 Klima H, Ullrich K, Aslanidis C, Fehringer P, Lackner KJ, Schmitz $\mathrm{G}$ : A splice junction mutation causes deletion of a 72-base exon from the mRNA for Iysosomal acid lipase in a patient with cholesteryl ester storage disease. J Clin Invest 1993; 92: 2713-2718.

21 Krawczak M, Reiss J, Cooper DN: The mutational spectrum of single base-pair substitutions in the mRNA splice junctions of human genes: causes and consequences. Hum Genet 1992; 90: 41-54.

22 O'Neill JP, Rogan PK, Cariello N, Nicklas JA: Mutations that alter RNA splicing of the human HPRT gene: a review of the spectrum. Mut Res 1998; 411: 179-214.

23 Bruyn RP, van Deutekom J, Frants RR, Padberg GW: Hereditary spastic paraparesis. Clinical and genetic data from a large Dutch family. Clin Neurol Neurosurg 1993; 95: 125-129.

24 Nielsen JE, Koefoed P, Abell K et al: CAG repeat expansion in autosomal dominant pure spastic paraplegia linked to chromosome2p21-p24. Hum Mol Genet 1997; 6: 1811-1816.

25 Schalling $M$, Hudson TJ, Buetow KH, Housman DE: Direct detection of novel expanded trinucleotide repeats in the human genome. Nat Genet 1993; 4: 135-139.

26 Dürr A, Brice A, Serdaru $M$ et al: The phenotype of 'pure' autosomal dominant spastic paraplegia. Neurology 1994; 44: 1274-1277.

27 Dürr A, Davoine CS, Paternotte C et al: Phenotype of autosomal dominant spastic paraplegia linked to chromosome2. Brain 1996; 119: 1487-1496. 